\title{
Simple Functors of Admissible Linear Categories
}

\author{
Laurence Barker' ${ }^{1}$ (D) Merve Demirel ${ }^{2}$
}

Received: 20 April 2015 / Accepted: 27 November 2015 / Published online: 14 January 2016

(C) Springer Science+Business Media Dordrecht 2016

\begin{abstract}
Generalizing an idea used by Bouc, Thévenaz, Webb and others, we introduce the notion of an admissible $R$-linear category for a commutative unital ring $R$. Given an $R$-linear category $\mathcal{L}$, we define an $\mathcal{L}$-functor to be a functor from $\mathcal{L}$ to the category of $R$-modules. In the case where $\mathcal{L}$ is admissible, we establish a bijective correspondence between the isomorphism classes of simple functors and the equivalence classes of pairs $(G, V)$ where $G$ is an object and $V$ is a module of a certain quotient of the endomorphism algebra of $G$. Here, two pairs $(F, U)$ and $(G, V)$ are equivalent provided there exists an isomorphism $F \leftarrow G$ effecting transport to $U$ from $V$. We apply this to the category of finite abelian $p$-groups and to a class of subcategories of the biset category.
\end{abstract}

Keywords Biset category · Group category $\cdot$ Seeds of simple functors · Quiver algebra of a linear category

Mathematics Subject Classification (2010) Primary: 20C20 · Secondary: 20J99

Presented by Radha Kessar.

This work was supported by Tübitak Scientific and Technological Research Funding Program 1001 under grant number 114F078.

Laurence Barker

barker@fen.bilkent.edu.tr

Merve Demirel

merve.demirel@bilkent.edu.tr

1 Department of Mathematics, Bilkent University, 06800 Bilkent, Ankara, Turkey

2 Department of Economics, Bilkent University, 06800 Bilkent, Ankara, Turkey 


\section{Introduction}

The theory of group functors involves linear categories whose objects are finite groups and whose morphisms include precursors of linear maps such as induction, restriction, conjugation, inflation. When we speak of a functor for such a category $\mathcal{L}$, we mean a functor from $\mathcal{L}$ to the category of modules of a coefficient ring. As an example, the operation sending a finite group to its character ring can be seen as a functor from the Mackey category to the category of $\mathbb{Z}$-modules.

There is a well-known technique, used in Thévenaz-Webb [7], [8, Section 2] and Bouc $[1,4.3 .10]$ for instance, which can sometimes be applied to classify the simple $\mathcal{L}$-functors. We shall abstract that technique, expressing it in the form of a general result, Theorem 2.4.

We shall give one application, Corollary 2.5 , lying outside the theory of group functors. Corollary 2.5 is a classification of the simple functors of the category of finite abelian $p$ groups.

Our main application of Theorem 2.4 concerns suitable subcategories of linear extensions of the biset category. Weakening a condition used by Bouc [1, 4.1.3], we shall introduce the notion of a group category with admissible factorizations. The weakening of the condition allows accommodation of some important examples, such as the biset version of the Mackey category associated with a fixed finite group. As we shall show in Theorem 3.7, given a group category $\mathcal{G}$ with admissible factorizations then, working with coefficients in any commutative unital ring $R$, the isomorphism classes of simple $R \mathcal{G}$-functors are parameterized by equivalence classes of pairs $(G, V)$, where $G$ is a group in $\mathcal{L}$ and $V$ is a simple module of the group algebra $\operatorname{Rout}_{\mathcal{G}}(G)$ of a certain subgroup $\operatorname{Out}_{\mathcal{G}}(G) \leq \operatorname{Out}(G)$.

A resemblance between Theorem 2.4 and Romero [5, 4.2] suggests the possibility of using Theorem 2.4 to classify the simple modules of suitable Green biset functors. The theorem cannot be applied, though, to all Green biset functors. Romero [6, Example 12] has shown that, letting $F$ be any field and letting $F B_{C_{4}}^{1}$ denote the Green biset functor over $F$ constructed from the monomial Burnside algebra with fibre group $C_{4}$, then the $F$ linear category $\mathcal{L}$ associated with $F B_{C_{4}}^{1}$ is not admissible and its simple modules cannot be parameterized in the manner described in the proof of [5, 4.2]. Indeed, $\mathcal{L}$ is non-admissible because $D_{8}$ and $Q_{8}$ are non-isomorphic in $\mathcal{L}$, yet [6, Example 12] exhibits $\mathcal{L}$-morphisms $\alpha: D_{8} \rightarrow Q_{8}$ and $\beta: Q_{8} \rightarrow D_{8}$ that do not factor through any group with order strictly dividing 8. Moreover, the composites $\alpha \beta$ and $\beta \alpha$ are idempotents. Letting $S$ be a simple $\mathcal{L}$-functor not annihilated by $\alpha \beta$, then $S$ is not annihilated by $\beta \alpha$, hence $S\left(D_{8}\right) \neq 0$ and $S\left(Q_{8}\right) \neq 0$, yet $S(G)=0$ for all groups of order strictly dividing 8 .

\section{Admissible Linear Categories}

Throughout, we let $R$ be a commutative unital ring. An $R$-linear category (also called an $R$-preadditive category) is defined to be a category such that the morphism sets are $R$ modules and the composition operation is $R$-bilinear. Functors between $R$-linear categories are understood to be $R$-linear in the sense of being $R$-linear on morphism sets. Plainly, an $R$-linear category $\mathcal{K}$ is small if and only if the class of objects $\operatorname{Obj}(\mathcal{K})$ is a set.

Let $\mathcal{L}$ be a small $R$-linear category. We define an $\mathcal{L}$-functor to be a functor from $\mathcal{L}$ to the category of $R$-modules. In this section, we shall construct two $R$-algebras such that the $\mathcal{L}$ functors can be identified with certain modules of those algebras. After discussing simple $\mathcal{L}$-functors in general, we shall introduce a condition on $\mathcal{L}$, called admissibility, under which we shall classify the simple $\mathcal{L}$-functors up to isomorphism. 
Given objects $F, G \in \operatorname{Obj}(\mathcal{L})$, we write $\mathcal{L}(F, G)$ to denote the $R$-module of $\mathcal{L}$-morphisms $F \leftarrow G$. As an algebra over $R$, we define the quiver algebra of $\mathcal{L}$ to be

$$
{ }^{\oplus} \mathcal{L}=\bigoplus_{F, G \in \operatorname{Obj}(\mathcal{L})} \mathcal{L}(F, G)
$$

equipped with the multiplication operation coming from composition of morphisms, products of incompatible morphisms being zero. Note that, to form the direct sum, we are making use of the hypothesis that $\mathcal{L}$ is small. Any element $x \in{ }^{\oplus} \mathcal{L}$ can be written uniquely in the form

$$
x=\sum_{F, G \in \operatorname{Obj}(\mathcal{L})}{ }_{F} x_{G}
$$

where each ${ }_{F} x_{G} \in \mathcal{L}(F, G)$ and only finitely many of the ${ }_{F} x_{G}$ are non-zero. We call ${ }_{F} x_{G}$ the $(F, G)$-entry of $x$. Using the notation ${ }_{F} x_{G} y_{H}={ }_{F} x_{G} \cdot{ }_{G} y_{H}$ for $x, y \in{ }^{\oplus} \mathcal{L}$ and $F, G, H \in \operatorname{Obj}(\mathcal{L})$, the $(F, H)$-entry of the product $x y$ is given by ${ }_{F}(x y)_{H}=\sum_{G F^{x}}{ }_{G} y_{H}$.

The quiver algebra ${ }^{\oplus} \mathcal{L}$ is unital if and only if $\operatorname{Obj}(\mathcal{L})$ is finite. We extend it to an algebra that is always unital, namely, the extended quiver algebra ${ }^{\Pi} \mathcal{L}$, whose elements $x$ have the same decomposition as before, $x=\sum_{F, G F} x_{G}$, but with a relaxation of the condition that only finitely many of the terms ${ }_{F} x_{G}$ are non-zero. Instead we impose the weaker condition that, for each object $F$, there are only finitely many objects $G$ such that ${ }_{F} x_{G} \neq 0$ or ${ }_{G} x_{F} \neq 0$. The formula for the multiplication operation on ${ }^{\Pi} \mathcal{L}$ is the same summation formula as before. The summation still makes sense because only finitely many of the terms are nonzero. We have equality ${ }^{\oplus} \mathcal{L}={ }^{\Pi} \mathcal{L}$ if and only if $\operatorname{Obj}(\mathcal{L})$ is finite.

The elements of ${ }^{\oplus} L$ can be viewed as matrices with rows and columns indexed by $\operatorname{Obj}(\mathcal{L})$, only finitely many of the matrix entries being non-zero. The elements of ${ }^{\Pi} \mathcal{L}$ can be viewed as matrices in a similar way, but with the finiteness condition now being that each row has only finitely many non-zero entries, likewise for each column.

With care, we can form infinite summations in ${ }^{\Pi} \mathcal{L}$. Let us say that a family $\left(x_{i}: i \in I\right)$ of elements $x_{i} \in{ }^{\Pi} \mathcal{L}$ is summable provided, for each $F \in \operatorname{Obj}(\mathcal{L})$, there are only finitely many $i$ and $G$ such that ${ }_{F}\left(x_{i}\right)_{G} \neq 0$ or ${ }_{G}\left(x_{i}\right)_{F} \neq 0$. For such a family, we define the sum $\sum_{i} x_{i} \in \Pi \mathcal{L}$ to be such that

$$
{ }_{F}\left(\sum_{i \in I} x_{i}\right)_{G}=\sum_{i \in I}{ }_{F}\left(x_{i}\right)_{G} .
$$

Plainly, we have a distributivity property, $x \sum_{i} x_{i}=\sum_{i} x x_{i}$ and similarly for $\left(\sum_{i} x_{i}\right) x$.

Let $\operatorname{id}_{G}$ denote the identity morphism on $G$. The hom-sets can be recovered from ${ }^{\oplus} \mathcal{L}$ and from ${ }^{\Pi} \mathcal{L}$ via the equality

$$
\mathcal{L}(F, G)=\operatorname{id}_{F} \cdot{ }^{\oplus} \mathcal{L} \cdot \operatorname{id}_{G}=\operatorname{id}_{F} \cdot{ }^{\Pi} \mathcal{L} \cdot \operatorname{id}_{G} .
$$

The family $\left(\operatorname{id}_{G}: G \in \operatorname{Obj}(\mathcal{L})\right)$ is summable and the unity element of ${ }^{\Pi} \mathcal{L}$ is the sum

$$
1_{\mathcal{L}}=\sum_{G \in \operatorname{Obj}(\mathcal{L})} \operatorname{id}_{G} .
$$

The next remark is obvious.

Remark 2.1 There are $R$-linear equivalences of categories between:

(a) the category of ${ }^{\oplus} \mathcal{L}$-modules $M_{\oplus}$ such that ${ }^{\oplus} \mathcal{L} . M_{\oplus}=M_{\oplus}$,

(b) the category of ${ }^{\Pi} \mathcal{L}$-modules $M_{\Pi}$ such that ${ }^{\oplus} \mathcal{L} . M_{\Pi}=M_{\Pi}$,

(c) the category of $\mathcal{L}$-functors $M$. 
The equivalences $M_{\oplus} \leftrightarrow M_{\Pi} \leftrightarrow M$ are such that, given one of the three objects $M_{\oplus}$ or $M_{\Pi}$ or $M$, then the other two are determined via the identifications $M_{\oplus}=M_{\Pi}=\bigoplus_{G} M(G)$ and $\operatorname{id}_{G} \cdot M_{\oplus}=\operatorname{id}_{G} \cdot M_{\Pi}=M(G)$.

Let us identify the three categories with each other, calling it the category of $\mathcal{L}$-functors. Plainly, it is an abelian category. We call $M(G)$ the coordinate module of $M$ at $G$. We usually think of an $\mathcal{L}$-functor $M$ as the direct sum of the coordinate modules, the action $M(F) \leftarrow M(G)$ of each morphism set $\mathcal{L}(F, G)$ extending to an action of ${ }^{\oplus} \mathcal{L}$ and further extending to an action of $\Pi \mathcal{L}$. Thus, the elements of $M$ can be viewed as column vectors with coordinates indexed by $\operatorname{Obj}(\mathcal{L})$, only finitely many of the coordinates being non-zero.

Proposition 2.2 Given a full $R$-linear subcategory $\mathcal{K}$ of $\mathcal{L}$, then restriction yields a bijective correspondence between the isomorphism classes of simple $\mathcal{K}$-functors and the isomorphism classes of those simple $\mathcal{L}$-functors which are not annihilated by restriction.

Proof The identity element of ${ }^{\Pi} \mathcal{K}$ is $1_{\mathcal{K}}=\sum_{G \in \operatorname{Obj}(\mathcal{K})} \operatorname{id}_{G}$. We have ${ }^{\Pi} \mathcal{K}=1_{\mathcal{K}} \cdot{ }^{\Pi} \mathcal{L} .1_{\mathcal{K}}$ as a subring of ${ }^{\Pi} \mathcal{L}$. The $\mathcal{L}$-functors not annihilated by restriction are precisely the $\mathcal{L}$-functors $M$ such that $1_{\mathcal{K}} . M \neq 0$. The assertion now follows from a theorem of Green [4, Section 6.2]: letting $i$ be an idempotent of a unital ring $A$, then the condition $S \cong i T$ characterizes a bijective correspondence between the isomorphism classes of simple $i A i$-modules $S$ and the isomorphism classes of simple $A$-modules $T$ such that $i T \neq 0$.

Observe that, given an object $G \in \operatorname{Obj}(\mathcal{L})$ and an $\mathcal{L}$-functor $M$ then, by restriction to the full subcategory with unique object $G$, the coordinate module $M(G)$ becomes a module of the algebra $L(G, G)=\operatorname{End}_{\mathcal{L}}(G)$. We define an ovule of $\mathcal{L}$ to be a pair $(G, V)$ where $G \in \operatorname{Obj}(\mathcal{L})$ and $V$ is a simple $L(G, G)$-module. Two ovules $(F, U)$ and $(G, V)$ of $\mathcal{L}$ are said to be equivalent provided there exist an $\mathcal{L}$-isomorphism $\alpha: F \leftarrow G$ and an $R$-isomorphism $\theta: U \leftarrow V$ which effect transport to the isomorphism class of $U$ from the isomorphism class of $V$, we mean,

$$
\rho_{U}\left(\alpha y \alpha^{-1}\right) \circ \theta=\theta \circ \rho_{V}(y)
$$

for all $y \in L(G, G)$, where $\rho_{U}$ and $\rho_{V}$ are the representations of $U$ and $V$, respectively.

Proposition 2.3 Let $(F, U)$ and $(G, V)$ be ovules of $\mathcal{L}$.

(1) There exists a simple $\mathcal{L}$-functor $S_{G, V}$, unique up to isomorphism, such that $S_{G, V}(G) \cong$ $V$.

(2) If $(F, U)$ and $(G, V)$ are equivalent, then $S_{F, U} \cong S_{G, V}$.

(3) If $F \cong_{\mathcal{L}} G$ but $(F, U)$ and $(G, V)$ are not equivalent, then $S_{F, U} ¥ S_{G, V}$.

Given a simple $\mathcal{L}$-functor $S$, letting $H$ be any object of $\mathcal{L}$ such that $S(H) \neq 0$ and defining $W=S(H)$, then $S \cong S_{H, W}$.

Proof This is the special case $\operatorname{Obj}(\mathcal{K})=\{G\}$ of Proposition 2.2.

The function sending the equivalence class of $(G, V)$ to the isomorphism classes of $S_{G, V}$ is surjective but not always injective. We shall now consider a condition on $\mathcal{L}$ which will allow us to assign a special status to some of the ovules of $\mathcal{L}$. Those ovules (the fertilized 
ones, it might be said) will give rise to pairs called seeds. We shall establish a bijective correspondence between the equivalence classes of seeds and the isomorphism classes of $\mathcal{L}$-functors.

A partial ordering is said to be well-founded provided it has no infinite descending chains. Let $\leq$ be a well-founded partial ordering relation on the set of isomorphism classes of objects of $\mathcal{L}$. Generalizing a condition in Bouc [1, 4.1.3], we say that $\mathcal{L}$ is admissible with respect to $\leq$ provided every morphism $x: F \leftarrow G$ in $\mathcal{L}$ can be expressed in the form

$$
x=\sum_{I} a_{I} b_{G}
$$

where ${ }_{F} a_{I} \in \mathcal{L}(F, I)$ and ${ }_{I} b_{G} \in \mathcal{L}(I, G)$ and $I$ runs over finitely many objects in $\mathcal{L}$ such that the isomorphism class $[I]$ satisfies $[F] \geq[I] \leq[G]$.

As an aside, let us mention that a non-admissible category may become admissible upon extention of coefficients. To see an example, let $\mathcal{N}$ be the $\mathbb{Z}$-linear category with three objects $x, y, z$ such that $\mathcal{L}(x, x), \mathcal{L}(y, y), \mathcal{L}(z, z), \mathcal{L}(x, y), \mathcal{L}(y, z), \mathcal{L}(x, z)$ are regular $\mathbb{Z}$ modules generated by $\operatorname{id}_{x}, \operatorname{id}_{y}, \operatorname{id}_{z}, \alpha, \beta, \gamma$ such that $\alpha \beta=2 \gamma$. Let $\leq$ be the partial ordering on the set of isomorphism classes $\{[x],[y],[z]\}$ such that only $[x]$ and $[y]$ are maximal elements and only $[y]$ is a minimal element. Then $\mathcal{N}$ is non-admissible because $\gamma$ does not factor through $[y]$. But the extension $\mathbb{Q N}$ is clearly admissible.

Suppose that $\mathcal{L}$ is admissible with respect to $\leq$. Given $G \in \operatorname{Obj}(\mathcal{L})$, we let $\mathcal{L}_{<}(G)$ denote the set of elements $x \in \mathcal{L}(G, G)$ that can be expressed as $x=\sum_{I} a_{I} b_{G}$ where ${ }_{G} a_{I} \in \mathcal{L}(G, I)$ and ${ }_{I} b_{G} \in \mathcal{L}(I, G)$ and $I$ runs over finitely many objects in $\mathcal{L}$ such that $[I]<[G]$. Plainly, $\mathcal{L}_{<}(G)$ is an ideal of $\mathcal{L}(G, G)$. As a unital algebra over $R$, we define

$$
\overline{\mathcal{L}}(G)=\mathcal{L}(G, G) / \mathcal{L}_{<}(G) .
$$

We define a seed for $\mathcal{L}$ to be a pair $(G, V)$ where $G \in \operatorname{Obj}(\mathcal{L})$ and $V$ is a simple $\overline{\mathcal{L}}(G)$ module. By inflation, we can regard $V$ as a simple $\mathcal{L}(G, G)$-module annihilated by $L_{<}(G)$. Hence, we can regard the seed $(G, V)$ as an ovule of $\mathcal{L}$. Two seeds of $\mathcal{L}$ are said to be equivalent provided they are equivalent as ovules.

The next theorem generalizes Thévenaz-Webb [8, Section 2] and Bouc [1, 4.3.10].

Theorem 2.4 Suppose that $\mathcal{L}$ is admissible with respect to a well-founded partial ordering on the isomorphism classes of $\mathcal{L}$. Then the condition $S \cong S_{G, V}$ characterizes a bijective correspondence between the isomorphism classes of simple $\mathcal{L}$-functors $S$ and the equivalence classes of seeds $(G, V)$ of $\mathcal{L}$. Furthermore, given a seed $(G, V)$, then the isomorphism class of $G$ is the minimum among the isomorphism classes of objects $F$ of $\mathcal{L}$ such that $S_{G, V}(F) \neq 0$.

Proof Given $S$ then, by the well-foundedness, there exists a minimal isomorphism class $[G]$ in $\mathcal{L}$ such that $S(G) \neq 0$. Letting $[F]$ be another minimal isomorphism class such that $S(F) \neq 0$ then, by the simplicity of $S$, there exist elements $x \in \mathcal{L}(F, G)$ and $s \in S(G)$ such that $x s \neq 0$. Writing $x=\sum_{I F} a_{I} b_{G}$ as above, with $[F] \geq[I] \leq[G]$, then ${ }_{I} b_{G} \cdot s \neq 0$ for some $I$, hence $S(I) \neq 0$. The minimality of $F$ and $G$ implies that $[F]=[I]=[G]$. We have shown that $[G]$ is unique. Letting $V=S(G)$ as an $\mathcal{L}(G, G)$-module, a similar argument shows that $V$ is annihilated by $\mathcal{L}_{<}(G)$. So $V$ can be regarded as a $\overline{\mathcal{L}}(G)$-module and $(G, V)$ is a seed. Invocation of Proposition 2.3 completes the argument. 
We end this section with an easy illustration of the main theorem. Let $p$ be a prime. Let $\mathcal{A}_{p}$ be the category of finite abelian $p$-groups. The next result, a variant of $[2,4.1 .3]$, is a classification of the simple $\mathcal{A}_{p}$-functors up to isomorphism.

Corollary 2.5 For each natural number $n$, there exists a simple $\mathcal{A}_{p}$-functor $S_{n}$, unique up to isomorphism, such that $A_{p}\left(C_{p^{n}}\right) \neq 0$. Every simple $\mathcal{A}_{p}$-functor is isomorphic to $S_{n}$ for some natural number $n$.

Proof Although $\mathcal{A}_{p}$ is not small, we can treat it as small by a universe-expanding argument or by replacing it with an equivalent small category. On the set of isomorphism classes in $\mathcal{A}_{p}$, we define a partial ordering $\leq$ such that, given finite abelian groups $F$ and $G$, then $[F] \leq[G]$ provided $F$ is isomorphic to a subquotient of $G$. In that case, of course, $F$ is isomorphic to a subgroup of $G$ and to a quotient group of $G$. Since every group homomorphism is the composite of a monomorphism and an epimorphism, $\mathcal{A}_{p}$ (or a small surrogate for $\mathcal{A}_{p}$ ) is admissible.

Fix $n$ and write $q=p^{n}$. For each integer $j$, let $\xi_{j}$ be the endomorphism of $C_{q}$ sending each element $a$ of $C_{q}$ to the element $a^{j}$. There is a ring isomorphism

$$
\mathcal{A}_{p}\left(C_{q}, C_{q}\right) \ni \xi_{j} \leftrightarrow j+q \mathbb{Z} \in \mathbb{Z} / q \mathbb{Z} .
$$

The endomorphism $\xi_{j}$ factors through a strict subquotient of $C_{q}$ if and only if $p$ divides $j$. So there is a ring isomorphism

$$
\overline{\mathcal{A}_{p}}\left(C_{q}\right) \ni \xi_{j}+\left(\mathcal{A}_{p}\right)_{<}\left(C_{q}\right) \leftrightarrow j+p \mathbb{Z} \in \mathbb{Z} / p \mathbb{Z} .
$$

Let $V_{q}$ denote the isomorphically unique simple $\overline{\mathcal{A}_{p}}\left(C_{q}\right)$-module. Let $S_{n}=S_{C_{q}, V_{q}}$. Since the inflation of $V_{q}$ is the isomorphically unique simple $\mathcal{A}_{p}\left(C_{q}, C_{q}\right)$-module, the rider of Proposition 2.3 already tells us that, given a simple $\mathcal{A}_{p}$-functor $S$ satisfying $S\left(C_{q}\right) \neq 0$, then $S \cong S_{n}$.

By Theorem 2.4, the simple $\mathcal{A}_{p}$-functors $S_{0}, S_{1}, \ldots$ are mutually non-isomorphic. It remains only to show that, given a simple $\mathcal{A}_{p}$-functor $S$, then $S \cong S_{n}$ for some $n$. Choose a finite abelian $p$-group $G$ such that $S(G) \neq 0$. Write $G=\prod_{i} G_{i}$ as a direct product of finitely many cyclic $p$-groups $G_{i}$. For each index $i$, let $v_{i}: G \leftarrow G_{i}$ be the inclusion and $\pi_{i}: G_{i} \leftarrow G$ the canonical projection. Choose an element $s \in S(G)-\{0\}$. Since $s=\sum_{i} v_{i}\left(\pi_{i}(s)\right)$, we have $\pi_{i}(s) \in S\left(G_{i}\right)-\{0\}$ for some $i$, hence $S\left(G_{i}\right) \neq 0$. Letting $n$ be the natural number such that $G_{i} \cong C_{p^{n}}$, then $S \cong S_{n}$.

\section{Group Categories with Admissible Factorizations}

In Theorem 3.7, an application of Theorem 2.4, we shall classify the simple functors for suitable subcategories of the $R$-linear extension of the biset category.

After Bouc [1, Chapters 2, 3], let us recall some features of the biset category $\mathcal{C}$. The class of objects $\operatorname{Obj}(\mathcal{C})$ is the class of finite groups. To describe the morphisms in $\mathcal{C}$, we shall need some notation. Consider finite groups $F, G, H$. The Burnside group $B(G)$ can be defined as the $\mathbb{Z}$-module freely generated by the isomorphism classes of the transitive $G$ sets. Isomorphism classes of $G$-sets are identified with elements of $B(G)$ in such a way that, given $G$-sets $Z$ and $Z^{\prime}$, writing $[Z]$ for the isomorphism class of $Z$, then $\left[Z \sqcup Z^{\prime}\right]=[Z]+\left[Z^{\prime}\right]$. 
An $F \times G$-set is called an $(F, G)$-biset. The set of morphisms $F \leftarrow G$ in $\mathcal{C}$ is defined to be $\mathcal{C}(F, G)=B(F \times G)$. Composition is defined to be the bilinear map

$$
\mathcal{C}(F, G) \times \mathcal{C}(G, H) \rightarrow \mathcal{C}(F, H)
$$

such that, given an $(F, G)$-biset $X$ and $(G, H)$-biset $Y$, then $[X][Y]=\left[X \times_{G} Y\right]$ where $X \times{ }_{G} Y$ denotes the $(F, H)$-biset of $G$-orbits of $X \times Y$. That completes the definition of $\mathcal{C}$ as a linear category. By replacing the universe with a larger one, we shall understand $\mathcal{C}$ to be small.

A morphism $\xi \in \mathcal{C}(F, G)$ is said to be transitive provided $\xi$ is the isomorphism class of a transitive $(F, G)$-biset. Any such $\xi$ has the form $\xi=[(F \times G) / I]$ where $I \leq F \times G$. Let $p_{1}(I)$ be the image of $I$ under the canonical projection $F \leftarrow F \times G$. Let $k_{1}(I)$ be the kernel of the canonical projection $G \leftarrow F \times G$. Define $p_{2}(I)$ and $k_{2}(I)$ similarly as subgroups of $G$. Define $q(I)=I /\left(k_{1}(I) \times k_{2}(I)\right)$. Given another subgroup $I^{\prime} \leq F \times G$ such that $\xi=\left[(F \times G) / I^{\prime}\right]$, then $I^{\prime}$ is conjugate to $I$ in $F \times G$. So, as an abstract group, $q(\xi)=q(I)$ is well-defined up to isomorphism. By Bouc [1, 2.3.21], the canonical homomorphisms $\sigma_{1}: p_{1}(I) / k_{1}(I) \leftarrow q(I)$ and $\sigma_{2}: p_{2}(I) / k_{2}(I) \leftarrow q(I)$ are isomorphisms. In particular, $q(\xi)$ is isomorphic to subquotients of $F$ and $G$.

We call $\xi$ a tinflation (or a transfer-inflation, sometimes also called an inductioninflation) provided $k_{2}(I)=1$ and $p_{2}(I)=G$. We call $\xi$ a destriction (or a deflationrestriction) provided $p_{1}(I)=F$ and $k_{1}(I)=1$. If there exists a tinflation $F \leftarrow G$, then $F$ has a subquotient isomorphic to $G$. Indeed, supposing that $\xi$ is a tinflation, then $p_{1}(I) / k_{1}(I) \cong G$. Similarly, if there exists a destriction $F \leftarrow G$, then $F$ is isomorphic to a subquotient of $G$. Every transitive morphism is the composite of a tinflation and a destriction. Indeed, defining

$$
\tau=\left[\frac{F \times q(I)}{\left\{\left(p_{1}(i), i\left(k_{1}(I) \times k_{2}(I)\right)\right): i \in I\right\}}\right], \quad \delta=\left[\frac{q(I) \times G}{\left\{\left(i\left(k_{1}(I) \times k_{2}(I)\right), p_{2}(i)\right): i \in I\right\}}\right]
$$

then $\tau$ is a tinflation, $\delta$ is a destriction and, by the formula for the product of two transitive morphisms in [1, 2.3.24], $\xi=\tau \delta$. We call $\xi$ an isogation provided $\xi$ is both a tinflation and a destriction. In that case, the groups $F$ and $G$ are isomorphic. Any group isomorphism $\rho: F \leftarrow G$, gives rise to an isogation

$$
{ }_{F} \text { iso }_{G}^{\rho}=[(F \times G) /\{(\rho(g), g): g \in G\}]
$$

and every isogation has that form. It is easy to see that, given group isomorphisms $\rho: F \leftarrow$ $G$ and $\sigma: G \leftarrow H$, then ${ }_{F}$ iso $_{G}^{\rho}$ iso $_{H}^{\sigma}={ }_{F}$ iso $_{H}^{\rho \sigma}$. The identity morphism on $G$ is the isogation ${ }_{G}$ iso $_{G}={ }_{G}$ iso $_{G}^{1}$. The next lemma is immediate from Bouc [1, 2.3.22, 2.3.24].

Lemma 3.1 (Bouc.) Given transitive morphisms $\xi: F \leftarrow G$ and $\eta: G \leftarrow H$, then there exist transitive morphisms $\zeta_{1}, \ldots, \zeta_{r}: F \leftarrow H$ such that $\xi \eta=\sum_{k} \zeta_{k}$. Furthermore, each $q\left(\zeta_{k}\right)$ is isomorphic to subquotients of $q(\xi)$ and $q(\eta)$.

We define a group category with inverses to be a linear subcategory $\mathcal{H}$ of $\mathcal{C}$ such that every morphism in $\mathcal{H}$ is a linear combination of transitive morphisms in $\mathcal{H}$ and, given an isogation ${ }_{F}$ iso $_{G}^{\rho}$ in $\mathcal{H}$, then the inverse isogation ${ }_{G}$ iso $_{F}^{\rho^{-1}}$ is in $\mathcal{H}$. Let $\mathcal{H}$ be a group 
category with inverses. We write $R \mathcal{H}$ to denote the $R$-linear category obtained by extension to coefficients in $R$. We mean to say, the $R$-module of morphisms $F \leftarrow G$ in $R \mathcal{H}$ is $R \mathcal{H}(F, G)=R \otimes_{\mathbb{Z}} \mathcal{H}(F, G)$.

Proposition 3.2 Given $F, G \in \operatorname{Obj}(\mathcal{H})$, then the following three conditions are equivalent:

(a) There exists an isogation $F \leftarrow G$ in $\mathcal{H}$.

(b) The groups $F$ and $G$ are isomorphic as objects of $\mathcal{H}$.

(c) The groups $F$ and $G$ are isomorphic as objects of $R \mathcal{H}$.

Proof It is trivial that (a) implies (b) and that (b) implies (c). Assume (c). Choose $x \in$ $R \mathcal{H}(F, G)$ and $y \in R \mathcal{H}(G, F)$ such that $x y={ }_{F}$ iso $_{F}$. Write $x=\sum_{i} a_{i} \xi_{i}$ and $y=\sum_{j} b_{j} \eta_{j}$ where the $\xi_{i}$ and $\eta_{j}$ are transitive morphisms and $a_{i}, b_{j} \in R$. There exist indices $i$ and $j$ such that, writing $\xi_{i} \eta_{j}=\sum_{k} \zeta_{k}$ as a sum of transitive morphisms, then $\zeta_{k}={ }_{F}$ iso $_{F}$ for some $k$. Lemma 3.1 implies that, as abstract groups, $F$ is isomorphic to a subquotient of $q\left(\xi_{i}\right)$. But $q\left(\xi_{i}\right)$ is isomorphic to a subquotient of $G$. Similarly, $G$ is isomorphic to a subquotient of $F$. So, still as abstract groups, $F$ and $q\left(\xi_{i}\right)$ and $G$ are mutually isomorphic. Therefore $\xi_{i}$ is an isogation.

Let $\operatorname{Aut}_{\mathcal{H}}(G)$ denote the subgroup of $\operatorname{Aut}(G)$ consisting of those automorphisms $\rho$ of $G$ such that ${ }_{G}$ iso $_{G}^{\rho} \in \mathcal{H}(F, G)$. Let $\operatorname{Aut}_{G}(G)$ denote the inner automorphism group of $G$. Let $\operatorname{Out}_{\mathcal{H}}(G)=\operatorname{Aut}_{\mathcal{H}}(G) / \operatorname{Aut}_{G}(G)$. We embed $\operatorname{Out}_{\mathcal{H}}(G)$ in the unit group of $R \mathcal{H}(G, G)$ such that, given $\rho \in \operatorname{Aut}_{\mathcal{H}}(G)$, then the image of $\rho$ in $\operatorname{Out}_{\mathcal{H}}(G)$ is identified with ${ }_{G}$ iso $_{G}^{\rho}$. The embedding extends $R$-linearly to an embedding of algebras $R \operatorname{Out}_{\mathcal{H}}(G) \hookrightarrow R \mathcal{H}(G, G)$. Let $R \mathcal{H}_{\neq}(G, G)$ be the ideal of $R \mathcal{H}(G, G)$ spanned by those transitive morphisms $\xi: G \leftarrow G$ such that $|q(\xi)|<|G|$. We shall make use of the following result of Bouc [1, 4.3.2].

Proposition 3.3 (Bouc.) Given $G \in \operatorname{Obj}(\mathcal{H})$, then $R \mathcal{H}(G, G)=R \operatorname{Out}_{\mathcal{H}}(G) \oplus R \mathcal{H}_{\neq}(G, G)$ as the direct sum of a subalgebra and an ideal.

We say that $\mathcal{H}$ has admissible factorizations provided every transitive morphism $\xi$ in $\mathcal{H}$ is the composite $\xi=\tau \delta$ of a tinflation $\tau$ in $\mathcal{H}$ and a destriction $\delta$ in $\mathcal{H}$.

The biset category $\mathcal{C}$ and, more generally, all the subcategories of $\mathcal{C}$ considered in Bouc's definition of "admissibility" [1, 4.1.3], are examples of group categories with admissible factorizations. To see an example not covered by his definition, let us fix a finite group $G$ and let $\mathcal{M}_{G}$ be the category whose objects are the subgroups of $G$ and whose morphisms are the $\mathbb{Z}$-linear combinations of the morphisms having the form $\left[(K \times L) /\left\{\left({ }^{g} j, j\right): j \in J\right\}\right]$ where $K \leq G \geq L \geq J$ and $g \in G$ with ${ }^{g} J \leq K$. It is easy to check that $\mathcal{M}_{G}$ is a group category with inverses and admissible factorizations. We mention that $\mathcal{M}_{G}$ is a quotient of a category discussed in Thévenaz-Webb [8] and every $\mathcal{M}_{G}$-functor is a Mackey functor in their sense. To see another example, let $\mathcal{F}$ be a fusion system on a finite $p$-group $P$ and let $\mathcal{M}_{\mathcal{F}}$ be the category whose objects are the subgroups of $P$ and whose morphisms are the $\mathbb{Z}$-linear combinations of the morphisms having the form $[(Q \times R) /\{(\phi(j), j): j \in J\}]$ where $Q \leq P \geq R \geq J$ and $\phi \in \operatorname{Hom}_{\mathcal{F}}(J, Q)$. Again, it is easy to show that $\mathcal{M}_{\mathcal{F}}$ is a group category with inverses and admissible factorizations. Every $\mathcal{M}_{\mathcal{F}}$-functor is a Mackey functor for $\mathcal{F}$ in the sense of Díaz-Park [3, 2.1].

Let $\mathcal{G}$ be a group category with inverses and admissible factorizations. Let $F, G \in$ $\operatorname{Obj}(\mathcal{G})$. We write $[G]_{\mathcal{G}}$ to denote the isomorphism class of $G$ in $R \mathcal{G}$. By Proposition 3.2, $[G]_{\mathcal{G}}$ does not depend on $R$. Let $\leq_{\mathcal{G}}$ be the minimal transitive relation on the isomorphism 
classes in $R \mathcal{G}$ such that, if there exists a tinflation $G \rightarrow F$ or a destriction $G \leftarrow F$, then $[G]_{\mathcal{G}} \leq_{\mathcal{G}}[F]_{\mathcal{G}}$.

Lemma 3.4 The relation $\leq_{\mathcal{G}}$ is a partial ordering.

Proof We must confirm that, if $[G]_{\mathcal{G}} \leq_{\mathcal{G}}[F]_{\mathcal{G}}$ and $[F]_{\mathcal{G}} \leq_{\mathcal{G}}[G]_{\mathcal{G}}$, then $[F]_{\mathcal{G}}=[G]_{\mathcal{G}}$. Let $H_{0}, \ldots, H_{r}$ be objects of $\mathcal{G}$ such that $H_{0}=G$ and $H_{r}=F$ and, for each $1 \leq i \leq r$, there is a morphism $\xi$ in $\mathcal{G}$ such that $\xi_{i}$ is a tinflation $H_{i-1} \rightarrow H_{i}$ or a destriction $H_{i-1} \leftarrow H_{i}$. Each $\left|H_{i-1}\right| \leq\left|H_{i}\right|$, so $|G| \leq|F|$. Similarly, $|G| \geq|F|$. Therefore $|G|=|F|$. It follows that each $\left|H_{i-1}\right|=\left|H_{i}\right|$, each $\xi_{i}$ is an isogation and the groups $H_{i}$ are mutually isomorphic in $R \mathcal{G}$.

Proposition 3.5 The $R$-linear category $R \mathcal{G}$ is admissible with respect to $\leq_{\mathcal{G}}$. Furthermore, given $G \in \operatorname{Obj}(\mathcal{G})$, then $R \mathcal{G}_{<}(G)=R \mathcal{G}_{\neq}(G, G)$ and $\overline{R \mathcal{G}}(G) \cong R \operatorname{Out}_{\mathcal{G}}(G)$.

Proof By the admissible factorization property of $\mathcal{G}$, every transitive morphism $F \leftarrow G$ in $\mathcal{G}$ factorizes through an object $Q$ such that $[Q]_{\mathcal{G}}$ is less than or equal to $[F]_{\mathcal{G}}$ and $[G]_{\mathcal{G}}$. So $R \mathcal{G}$ is admissible with respect to $\leq_{G}$. The admissible factorization property also implies that $R \mathcal{G}_{<}(G)=R \mathcal{G}_{\neq}(G, G)$. An appeal to Proposition 3.3 completes the argument.

In view of the latest proposition, we can understand a seed of $R \mathcal{G}$ to be a pair $(G, V)$ where $G \in \operatorname{Obj}(\mathcal{G})$ and $V$ is a simple $R \operatorname{Out}_{\mathcal{G}}(G)$-module. Let us check that, in this context, the equivalence relation on seeds as we defined it in the previous section coincides with the equivalence relation defined by Bouc [1, 4.3.6].

Remark 3.6 Two seeds $(F, U)$ and $(G, V)$ of $R \mathcal{G}$ are equivalent if and only if there exist $\sigma \in \operatorname{Iso}_{\mathcal{G}}(F, G)$ and an $R$-isomorphism $\phi: U \leftarrow V$ such that, given $\epsilon \in \operatorname{Aut}_{\mathcal{G}}(G)$, then

$$
\rho_{U}\left({ }_{F} \text { iso }_{F}^{\sigma \epsilon \sigma^{-1}}\right) \circ \phi=\phi \circ \rho_{V}\left({ }_{G} \text { iso }_{G}^{\epsilon}\right) \text {. }
$$

Proof Suppose that $(F, U)$ and $(G, V)$ are equivalent. Choose an $R \mathcal{G}$-isomorphism $\alpha$ : $F \leftarrow G$ and an $R$-isomorphism $\theta: U \leftarrow V$ effecting transport from $U$ to $V$ in the sense specified in Section 2. By Proposition 3.2, there exists an isomorphism $\sigma: F \leftarrow G$ such that ${ }_{F}$ iso $_{G}^{\sigma} \in \mathcal{G}(F, G)$. Let $\beta=\alpha^{-1} \circ{ }_{F}$ iso $_{G}^{\sigma}$, which is a unit in $R \mathcal{G}(G, G)$. Let $\phi=\theta \circ \rho_{V}(\beta)$. We have

$$
\begin{gathered}
\rho_{U}\left({ }_{F} \text { iso }_{F}^{\sigma \epsilon \sigma^{-1}}\right) \circ \phi=\rho_{U}\left(\alpha \circ \beta \circ{ }_{G} \text { iso }_{G}^{\epsilon} \circ \beta^{-1} \circ \alpha^{-1}\right) \circ \theta \circ \rho_{V}(\beta) \\
=\theta \circ \rho_{V}\left(\beta \circ{ }_{G} \text { iso }_{G}^{\epsilon} \circ \beta^{-1}\right) \circ \rho_{V}(\beta)=\phi \circ \rho_{V}\left({ }_{G} \text { iso }_{G}^{\epsilon}\right) .
\end{gathered}
$$

The converse is trivial.

The next theorem is another generalization of Bouc [1, 4.3.10].

Theorem 3.7 The condition $S \cong S_{G, V}$ characterizes a bijective correspondence between the isomorphism classes of simple $R \mathcal{G}$-functors $S$ and the equivalence classes of seeds $(G, V)$ of $\mathcal{G}$. Furthermore, given $F \in \operatorname{Obj}(\mathcal{G})$ such that $S_{G, V}(F) \neq 0$ then, as an abstract group, $G$ is isomorphic to a subquotient of $F$. 
Proof This follows immediately from Theorem 2.4 and Proposition 3.5.

Acknowledgments The authors would like to thank Robert Boltje for some stimulating discussions which lay behind this work.

\section{References}

1. Bouc, S.: Biset Functors for Finite Groups. Lecture Notes in Math, vol. 1990. Springer, Berlin (2010)

2. Demirel, M.: Simple functors of admissible linear categories, MS thesis. Bilkent University, Ankara (2013)

3. Díaz, A., Park, S.: Mackey functors and sharpness of fusion systems. Homology, Homotopy Appl. 17, 147-164 (2015)

4. Green, J.A.: Polynomial Representations of $G L_{n}$. 2nd edn., Lecture Notes in Math, vol. 830. Springer, Berlin (2007)

5. Romero, N.: Simple modules over Green biset functors. J. Algebra 367, 203-221 (2012)

6. Romero, N.: On fibred biset functors with fibres of order prime and four. J. Algebra 387, 185-194 (2013)

7. Thévenaz, J., Webb, P.: Simple Mackey functors. In: Proceedings of 2nd International Group Theory Conference, Bressanone (1989), Supplement to Rendiconti del Circolo Matematico di Palermo, vol. 23, pp. 299-319 (1990)

8. Thévenaz, J., Webb, P.J.: The structure of Mackey functors. Trans. Amer. Math. Soc 347, 1865-1961 (1995) 